\title{
On the parameterized complexity of 2-partitions*
}

\author{
J. B. Andersen ${ }^{\dagger} \quad$ J. Bang-Jensen ${ }^{\ddagger} \quad$ A. Yeo ${ }^{\S}$
}

March 17, 2020

\begin{abstract}
We give an FPT algorithm for deciding whether the vertex set a digraph $D$ can be partitioned into two disjoint sets $V_{1}, V_{2}$ such that the digraph $D\left[V_{1}\right]$ induced by $V_{1}$ has a vertex that can reach all other vertices by directed paths, the digraph $D\left[V_{2}\right]$ has no vertex of in-degree zero and $\left|V_{i}\right| \geq k_{i}$, where $k_{1}, k_{2}$ are part of the input. This settles an open problem from [1, 4].
\end{abstract}

Keywords: FPT algorithm, out-branching, 2-partition, directed graph, parameterized complexity.

\section{Introduction}

A 2-partition of a digraph $D=(V, A)$ is a partition $\left(V_{1}, V_{2}\right)$ of $V$ into disjoint sets. Let $\mathcal{P}_{1}, \mathcal{P}_{2}$ be two (di)graph properties. Then a $\left(\mathcal{P}_{1}, \mathcal{P}_{2}\right)$-partition of a digraph $D$ is a 2-partition $\left(V_{1}, V_{2}\right)$ so that $D\left[V_{i}\right]$ has property $\mathcal{P}_{i}$ for $i=1,2$, where $D\left[V_{i}\right]$ is the subdigraph induced by $V_{i}$. A $\left(\mathcal{P}_{1}, \mathcal{P}_{2}\right)-\left[k_{1}, k_{2}\right]$-partition of a digraph is as above, but now we also require that $\left|V_{i}\right| \geq k_{i}$ for $i=1,2$. For example if $\mathcal{P}$ is the property of being acyclic, then the set of digraphs that allow a $(\mathcal{P}, \mathcal{P})$-partition are exactly those digraphs that have dichromatic number at most 2. Recognizing such digraphs is NP-complete [6].

Problems concerning the existence of certain 2-partitions of a given input digraph has received a lot of attention in the literature, see e.g. [1, 3, 4, 5, , 7, 8, 9, 10, 12, 13, 14]. In the papers [1, 3] the authors gave, for fixed $k_{1}, k_{2}$ (not part of the input), a complete complexity classification, in terms of being NP-complete or in XP, for the $120\left(\mathcal{P}_{1}, \mathcal{P}_{2}\right)-$ $\left[k_{1}, k_{2}\right]$-partition problems corresponding to properties $\mathcal{P}_{1}, \mathcal{P}_{2}$ both being one of the following 15 properties: being strongly connected, being connected, minimum outdegree at least 1 , minimum in-degree at least 1 , minimum in- and out-degree at least

*Research supported by the Independent Research Fond Denmark under grant number DFF 701400037B.

${ }^{\dagger}$ Department of Mathematics and Computer Science, University of Southern Denmark, Odense, Denmark (email: jonan15@student.sdu.dk)

${ }^{\ddagger}$ Department of Mathematics and Computer Science, University of Southern Denmark, Odense, Denmark (email: jbj@imada.sdu.dk)

${ }^{\S}$ Department of Mathematics and Computer Science, University of Southern Denmark, Odense, Denmark (email: yeo@imada.sdu.dk) 
1, minimum degree at least 1 , having an out-branching, having an in-branching, being acyclic, being complete, being oriented, being independent, being semicomplete, being a tournament and finally being symmetric. They left open to characterize which of those problems that are polynomial actually admit an FPT algorithm. In [4 this was settled for the 23 polynomially solvable problems coming from both $\mathcal{P}_{1}$ and $\mathcal{P}_{2}$ being one of the following 8 properties: strongly connected, connected, having an out-branching, having an in-branching, having minimum degree at least 1, having minimum in- and out-degree at least 1 , being acyclic, being complete. One problem that was left open in [4] was to determine the parameterized complexity of deciding the existence of a 2-partition $\left(V_{1}, V_{2}\right)$ with $\left|V_{i}\right| \geq k_{i}$ for $i=1$, 2 where the digraph $D\left[V_{1}\right]$ induced by $V_{1}$ has an out-branching and the digraph $D\left[V_{2}\right]$ has no vertex of in-degree zero. We prove in this paper that this problem is FPT.

\section{Notation and preliminaries}

The notation we use is consistent with that of [2]. For a digraph $D=(V, A)$ we say that two vertices $u$ and $v$ are adjacent if at least one of the arcs $u v, v u$ is in $A$. For a set of vertices $U \subseteq V$, the subdigraph induced by $U$, denoted $D[U]$, is the digraph obtained from $D$ by deleting all vertices $V \backslash U$ and all arcs adjacent to those vertices. For digraph $D$ we denote by $|D|=|V(D)|$ the number of vertices in the graph. We use the same notation for paths and cycles, so $|P|$ is the number of vertices in the path $P$. Paths and cycles will always be directed. The girth of a digraph is the length of a shortest directed cycle in $D$. A $(u, v)$-path is a directed path from $u \in V$ to $v \in V$ and the digraph $D$ is strongly connected if it contains a $(u, v)$-path for all ordered pairs of vertices $u, v \in V$. A strong component of a digraph is a maximal induced subdigraph which is strong. A strong component is initial if it has no entering arc in $D$.

The underlying graph, $U(D)$, of a digraph $D$ is the graph obtained from $D$ by replacing every 2-cycle by one edge and then suppressing all the directions of the other arcs. A digraph $D$ is connected if $U(D)$ is connected. For a vertex $u \in V$, we denote by $N(u)$ its neighbours, that is, the set of vertices that are adjacent to $u$. The outdegree $d_{D}^{+}(u)$ is the number of arcs going out of $u$ in $D$. Similarly, the in-degree $d_{D}^{-}(u)$ is the number of arcs going into $u$. We denote by $\delta^{-}(D)$ the minimum in-degree of a vertex in $D$. For a subset $X \subset V, N^{+}(X)$ denotes the set of out-neighbours of $X$ in $D$.

An out-tree rooted in $s$ is a connected digraph $T_{s}^{+}$such that $d_{T_{s}^{+}}^{-}(s)=0$ and $d_{T_{s}^{+}}^{-}(u)=1$ for all $u \in V\left(T_{s}^{+}\right) \backslash\{s\}$. An out-branching, $B^{+}$, of a digraph $D$ is an out-tree such that $V\left(T_{s}^{+}\right)=V(D)$.

A digraph $D$ is acyclic if it contains no induced directed cycles, and it is complete if for every pair of vertices $u, v \in V$ induce a 2-cycle $u v u$.

A parameterized problem with parameter $k$ is in the complexity class XP if instances of size $n$ can be solved in time $O\left(f(k) n^{g(k)}\right)$ for some pair of computable functions $f, g$. So if $k$ is fixed the problem can be solved in polynomial time. A problem is Fixed Parameter Tractable (FPT) if it can be solved in time $O\left(f(k) n^{c}\right)$ for some constant $c$ and computable function $f$. 


\section{The $\left(B^{+}, \delta^{-} \geq 1\right)-\left[k_{1}, k_{2}\right]$-partition problem}

The following is the problem whose complexity status we settle in this paper.

$\left(B^{+}, \delta^{-} \geq 1\right)-\left[k_{1}, k_{2}\right]$-PARTITION

Input: A digraph $D=(V, A)$ and natural numbers $k_{1}, k_{2}$

Question: Is there a 2-partition $\left(V_{1}, V_{2}\right)$ of $V$ such that $D\left[V_{1}\right]$ has an out-branching and $\delta^{-}\left(D\left[V_{2}\right]\right) \geq 1$, where $\left|V_{i}\right| \geq k_{i}$ for $i=1,2$ ?

In [1] the problem was shown to be polynomially solvable for every fixed pair of natural numbers $k_{1}, k_{2}$, but the algorithm has a running time $O\left(n^{f\left(k_{1}\right)}\right)$ and hence is not an FPT algorithm.

We begin with a few simple observations. The root of the out-branching is the only vertex that can possibly have in-degree 0 in a yes-instance. Therefore, if there are two or more vertices with in-degree 0 in $D$, it must be a no-instance. So in the following we assume the input is a digraph with at most one vertex with in-degree 0 . For future reference, if there is such a vertex with in-degree 0 in the input $D$, we will refer to it as $r$, and in that case $r$ must be the root of the out-branching.

Throughout the solution to the $\left(B^{+}, \delta^{-} \geq 1\right)-\left[k_{1}, k_{2}\right]$-partition problem, we will say that we grow some subset of the vertices inside the graph. What we mean by grow is iteratively adding a vertex to the set that is an out-neighbour of a vertex in the set. Sometimes we want to limit this process to only growing the set to a certain size. We formalize the process in Algorithm 1 ,

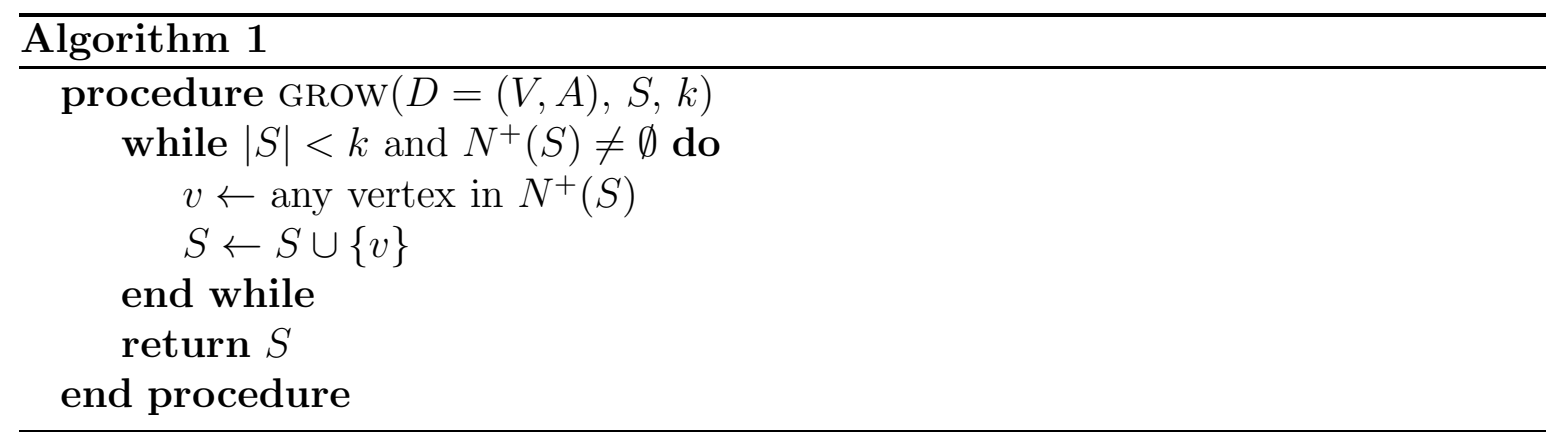

Note that if we don't want to limit the growth we can set $k=|V|$.

Lemma 1. Algorithm 1 runs in polynomial time.

Proof. Calculating the set of out-neighbours of a set can be done in polynomial time. A vertex is added to $S$ in each iteration of the while loop, so if none of the exit conditions are met before the $(n-1)$ st iteration, then after that iteration every vertex in the graph has been added to $S$ and thus its neighbourhood must be empty. So the algorithm runs in polynomial time.

If $\min \left\{k_{1}, k_{2}\right\}=0$, then we can solve the $\left(B^{+}, \delta^{-} \geq 1\right)$-[ $\left[k_{1}, k_{2}\right]$-PARTITION problem in polynomial time. If $k_{2}=0$ we can try all possible roots of the out-branching (there is only one possible root if $r$ exists), and grow it as large as possible by using Algorithm 
1, with $S$ containing the single vertex $s$ that we are trying as root and $k=|V|$. As we start with a single vertex and each vertex that is added has at least one arc into it from the previous set, it follows by induction that the subdigraph $D\langle S\rangle$ induced by the set $S$ returned by Algorithm 1 will contain an out-branching $T_{s}^{+}$. If one of the possible roots grows to a set $S$ with $|S| \geq k_{1}$, then because none of the vertices in $S$ has arcs to vertices in $V \backslash S$, it follows from our assumption that every vertex of $V$ (except possibly $r$ ) has in-degree at least 1 , that we have $\delta^{-}(D-S) \geq 1$. Thus, $\left(V_{1}, V_{2}\right)=(S, V \backslash S)$ is a solution.

For the case $k_{1}=0$, we will use the following algorithm which will prove useful later as well.

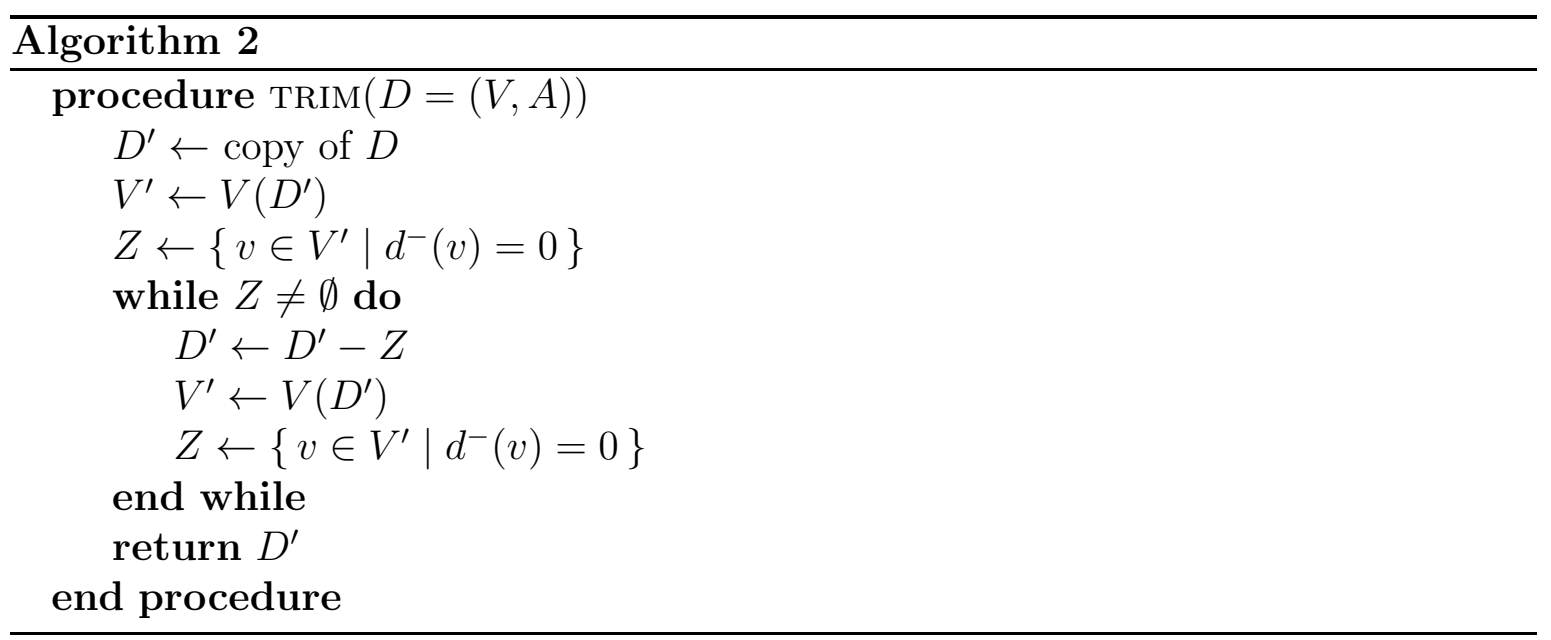

Algorithm 2 simply iteratively removes vertices with in-degree 0 in the current digraph and return the resulting digraph. Note that the resulting digraph $D^{\prime}$ will have $\delta^{-}\left(D^{\prime}\right) \geq 1$.

Lemma 2. Algorithm 2 finds the largest possible subdigraph with $\delta^{-} \geq 1$ in polynomial time.

Proof. It is clear that the algorithm runs in polynomial time and the other part of the claim follows from the fact that a vertex is only removed if it cannot be part of any subdigraph of minimum in-degree at least 1.

To handle the case $k_{1}=0$ we simply apply Algorithm 2 to the input $D$. Note that the removed vertices (if any) induce a subdigraph with an out-branching rooted at $r$, because a vertex only has in-degree 0 if it is $r$ or if its in-neighbours were removed, implying that an arc into it existed in $D$. Hence if the output $D^{\prime}=\left(V^{\prime}, A^{\prime}\right)$ of has $\left|V^{\prime}\right| \geq k_{2}$ then $\left(V \backslash V^{\prime}, V^{\prime}\right)$ is a solution.

From now on we assume that $k_{1}, k_{2} \geq 1$. Let us call a 2-partition $\left(V_{1}, V_{2}\right)$ of $V$ good if $D\left[V_{1}\right]$ has an out-branching and $\delta^{-}\left(D\left[V_{2}\right]\right) \geq 1$. As we have assumed that we have at most one vertex with in-degree 0 , the following lemma, which is the basis of the polynomial algorithm of Theorem 3.9 in [1], shows that in order to verify that $\left(D, k_{1}, k_{2}\right)$ is a 'yes'-instance we only need to find an induced subdigraph $D^{\prime}$ of $D$ such that $\left(D^{\prime}, k_{1}, k_{2}\right)$ is a 'yes'-instance. 
Lemma 3. Let $I=\left(D=(V, A), k_{1}, k_{2}\right)$ be an instance of the $\left(B^{+}, \delta^{-} \geq 1\right)-\left[k_{1}, k_{2}\right]$ partition problem with at most 1 vertex with in-degree 0 . Then any 2-partition $\left(V_{1}^{\prime}, V_{2}^{\prime}\right)$ (with $r \in V_{1}^{\prime}$ if $r$ exists) of a subset of $V$, where

- $D\left[V_{1}^{\prime}\right]$ has an out-branching

- $\delta^{-}\left(D\left[V_{2}^{\prime}\right]\right) \geq 1$

- $\left|V_{i}^{\prime}\right| \geq k_{i}, i=1,2$

can be extended to a good partition of $V$ in polynomial time.

Proof. Call a 2-partition $\left(V_{1}^{\prime}, V_{2}^{\prime}\right)$ that satisfies the conditions of the lemma a subsolution.

Fix any subsolution. Use Algorithm 1 on the graph $D-V_{2}^{\prime}$, starting with $S=V_{1}^{\prime}$ and $k=\left|D-V_{2}^{\prime}\right|$, and denote the result by $V_{T^{+}}$. In other words we grow the out-branching as large as possible, while not using any vertex in $V_{2}^{\prime}$. We claim that $\left(V_{T^{+}}, V \backslash V_{T^{+}}\right)$is a solution. Clearly $\left|V_{T^{+}}\right| \geq\left|V_{1}^{\prime}\right| \geq k_{1}$. Since $D\left[V_{1}^{\prime}\right]$ has an out-branching and Algorithm 1 only adds a vertex $v$ if it has an in-neighbour in the current set $S, V_{T^{+}}$must contain an out-branching. Secondly, because $r \in V_{1}^{\prime} \subseteq V_{T^{+}}$if it exists, we know that all vertices in $V_{2}=V \backslash V_{T^{+}}$had in-degree at least 1 in $D$. It was also the case that $\delta^{-}\left(D\left[V_{2}^{\prime}\right]\right) \geq 1$. Now because $V_{T^{+}}$was grown as large as possible in $D-V_{2}^{\prime}$, the vertices in $V_{2} \backslash V_{2}^{\prime}$ (if any) were not reachable from $V_{T^{+}}$and thus must still have in-degree at least 1 in $D\left[V_{2}\right]$. Moreover, $V_{2}^{\prime} \subseteq V_{2}$ meaning $\left|V_{2}\right| \geq\left|V_{2}^{\prime}\right| \geq k_{2}$, and thus $\left(V_{T^{+}}, V_{2}\right)$ is a solution.

As this is a simple application of Algorithm 1 a subsolution can be extended to a solution in polynomial time.

So from here on, finding a subsolution is sufficient to solve the problem.

The following result, due to Shen, will be used in the proof of our main result.

Theorem 4. [11] Suppose $G$ is a digraph of order $n$ and girth $g$ with $\delta^{+}(G) \geq 1$. Let $t=\left|\left\{u \in G \mid d^{+}(u)=1\right\}\right|$. Then

$$
g \leq \begin{cases}\lceil n / 2\rceil & \text { if } t=0, \\ \lceil(n+t-1) / 2\rceil & \text { if } t \geq 1\end{cases}
$$

The result also holds if we let $t$ be the number of vertices with in-degree 1 (in a digraph with $\delta^{-}(G) \geq 1$ ), instead of out-degree 1 (just reverse all arcs and apply Theorem (4). We are now ready to prove our main result.

Theorem 5. The $\left(B^{+}, \delta^{-} \geq 1\right)-\left[k_{1}, k_{2}\right]$-partition problem is FPT.

Proof. We will describe an FPT algorithm which correctly decides whether the input $\left(D, k_{1}, k_{2}\right)$ is a yes-instance of the $\left(B^{+}, \delta^{-} \geq 1\right)-\left[k_{1}, k_{2}\right]$-partition problem.

From the previous observations, we can assume that the input $\left(D, k_{1}, k_{2}\right)$ satisfies that $\min \left\{k_{1}, k_{2}\right\} \geq 1$ and $D$ has at most one vertex with in-degree 0 . Let $k=\max \left(k_{1}, k_{2}\right)$ and define the functions $f, h$ by setting $f(k)=32 k^{3}+4 k$ and 
$h(k)=2 k \cdot f(k)$.

We start by determining some arcs that cannot be part of the out-branching in any solution. We say that an arc $u v \in A$ is non-branchable if $D-\{u, v\}$ does not have a subdigraph $D^{\prime \prime}$ of size at least $k_{2}$ with $\delta^{-}\left(D^{\prime \prime}\right) \geq 1$. Thus if an arc $u v$ is non-branchable, then at least one of the vertices $u, v$ must belong to $V_{2}$ in any good partition $\left(V_{1}, V_{2}\right)$. Using Algorithm 2 we can identify the set $B^{\dagger} \subset A$ of non-branchable arcs in polynomial time. Let $B=A \backslash B^{\dagger}$ be the potential branching arcs and let $D_{B}=(V, B)$ be the subdigraph of $D$ containing exactly the arcs in $B$. For each vertex $u \in V$ we now define $N_{B}^{+}(u)$ as the out-neighbours of $u$ in $D_{B}$.

$$
N_{B}^{+}(u)=N_{D_{B}}^{+}(u)=\{v \mid u v \in B\}
$$

Clearly, $N_{B}^{+}(u)$ can be calculated for each $u \in V$ in polynomial time. We now distinguish several cases which cover all the possibilities.

Case 1. $\forall u \in V:\left|N_{B}^{+}(u)\right| \leq h(k)$ :

In this case we can check all possible out-trees of size exactly $k_{1}$ in $D_{B}$. We do this by trying each possible root, of which there are at most $n=|V|$ (there is only 1 if $r$ exists), and for each root the out-tree will have height at most $k_{1}$.

For each vertex, already included in the out-tree, we can use between 0 and $k_{1}$ of at most $h(k)$ different arcs leaving that vertex. There are at most

$$
\sum_{i=0}^{k_{1}}\left(\begin{array}{c}
h(k) \\
i
\end{array}\right)
$$

ways to do so.

So a very rough upper bound on the total number of out-trees that must be checked is

$$
n \cdot\left(\sum_{i=0}^{k_{1}}\left(\begin{array}{c}
h(k) \\
i
\end{array}\right)\right)^{k_{1}}
$$

which means if each check can be done in polynomial time, we can solve this case in FPT time.

For each possible out-tree $T^{+}$with $k_{1}$ vertices we run Algorithm 2 on $D-V\left(T^{+}\right)$. If the resulting graph $D^{\prime}$ has at least $k_{2}$ vertices, then $\left(V_{1}^{\prime}, V_{2}^{\prime}\right)=\left(V\left(T^{+}\right), V\left(D^{\prime}\right)\right)$ is a subsolution. As Algorithm 2 is indeed a polynomial time algorithm, we conclude that Case 1 can be solved in FPT-time.

For the case where $\max _{u \in V}\left\{\left|N_{B}^{+}(u)\right|\right\}>h(k)$, we first split the case into whether or not the vertex $r$ exists, and begin with the case where $r$ does not exist.

Case 2. $\delta^{-}(D) \geq 1$ and $\exists s \in V:\left|N_{B}^{+}(s)\right|>h(k)$ :

We will show that, if we are in this case then $\left(D, k_{1}, k_{2}\right)$ is a yes-instance and, in fact, any vertex $s$ with $\left|N_{B}^{+}(s)\right|>h(k)$, can be the root of the out-branching in a solution.

Fix any $s$ with $\left|N_{B}^{+}(s)\right|>h(k)$. 
We will say that we contract $v$ into $u$, if the arc $u v$ exists. Contracting $v$ into $u$ means removing the vertex $v$ and adding arcs from $u$ to every out-neighbour $w$ of $v$ (if $u w$ is not already an arc). As it turns out, we will only contract vertices into our choice for the root of the out-branching $s$, and unless otherwise stated, we do not rely on the vertices that are contracted into $s$ for our solution.

Let $\mathscr{C}$ be the set of strong components of $D-s$ and for each strong component $C \in \mathscr{C}$ let $N_{B, C}^{+}(s)$ denote the set of out-neighbours of $s$ inside $C$ using arcs in $B$, that is,

$$
N_{B, C}^{+}(s)=N_{B}^{+}(s) \cap V(C)
$$

Note that $\mathscr{C}$ and $N_{B, C}^{+}(s)$ can easily be computed in polynomial time.

Case 2.1. $\exists C \in \mathscr{C}:\left|N_{B, C}^{+}(s)\right| \geq f(k)$ :

Fix $C \in \mathscr{C}$ such that $\left|N_{B, C}^{+}(s)\right| \geq f(k)$. First, we look for a subsolution $\left(V_{1}^{\prime}, V_{2}^{\prime}\right)$, where $C$ is contained completely in one of the sets $V_{i}^{\prime}$.

Let $D^{\prime}$ be the output from Algorithm 2 on input $D-C-s$ (i.e. $D^{\prime}$ is a maximum subdigraph in $D-C-s$ with $\left.\delta^{-}\left(D^{\prime}\right) \geq 1\right)$. If $\left|D^{\prime}\right| \geq k_{2}$, then $\left(\{s\} \cup V(C), V\left(D^{\prime}\right)\right)$ is a subsolution, as $s$ has at least $f(k) \geq k_{1}$ out-neighbours in $C$ and $\delta^{-}\left(D^{\prime}\right) \geq 1$. Similarly, we can try Algorithm 1 on $D_{B}-C$ with $S=\{s\}$ and $k=k_{1}$. If the returned vertex set $V_{1}^{\prime}$ has size $\left|V_{1}^{\prime}\right| \geq k_{1}$, then, because $C$ is a strong component with at least $f(k) \geq k_{2}$ vertices, $\left(V_{1}^{\prime}, V(C)\right)$ is a subsolution. Both cases can clearly be handled in polynomial time so we may assume that neither of these cases occur, implying that we must split $C$ in some way to obtain a (sub)solution.

Iteratively contract all trivial initial strong components into $s$ (iteratively these are the vertices with in-degree 0 in the current digraph, $D-s$.) and call the resulting digraph $D$ for simplicity. If $C$ is not an initial strong component of $D$ after this process, then there is a non-trivial strong component $C^{\prime}$ with a path into $C$. Using Algorithm 1 on $D-s$ with $S=V\left(C^{\prime}\right)$ and $k=k_{2}$, we obtain a set $V_{2}^{\prime}$, where $\delta^{-}\left(D\left[V_{2}^{\prime}\right]\right) \geq 1$ because we started with a non-trivial strong component. We also have $\left|V_{2}^{\prime}\right| \geq k_{2}$ and $\left|V_{2}^{\prime} \cap V(C)\right|<k_{2}$ thus $V_{1}^{\prime}=N_{B, C}^{+}(s) \backslash V_{2}^{\prime}$ has size $\left|V_{1}^{\prime}\right| \geq f(k)-k_{2} \geq k_{1}$, so $\left(V_{1}^{\prime} \cup\{s\}, V_{2}^{\prime}\right)$ is a subsolution which can be found in polynomial time. Hence we can assume that $C$ is an initial strong component after the contraction step.

Now, observe that any cycle in $D[V(C)]$, small or large, that avoids at least $k_{1}$ vertices of $N_{B, C}^{+}(s)$, gives rise to a subsolution. Clearly, if such a cycle $O$ contains at least $k_{2}$ vertices, we immediately have a subsolution by taking an out-star consisting of $s$ and $k_{1}-1$ vertices from $N_{B, C}^{+}(s)-O$ for $V_{1}^{\prime}$ and the vertices of $O$ for $V_{2}^{\prime}$. If $O$ contains less than $k_{2}$ vertices, then we can grow it until it has $k_{2}$ vertices, which again leaves at least $f(k)-k_{2} \geq k_{1}$ vertices for an out-branching from $s$ (we can just take an out-star from $s$ with $k_{1}-1$ leaves). Hence it suffices to show that such a cycle $O$ indeed exists.

We first check whether $D\left[V(C)-N_{B, C}^{+}(s)\right]$ is acyclic. If this is not the case, then by the previous observation we have a subsolution and we are done. The same conclusion holds if there is a cycle in $C$ which contains only one vertex of $N_{B, C}^{+}(s)$ (and the 
existence of such a cycle can easily be checked in polynomial time). Hence we may assume that every cycle in $C$ contains at least two vertices of $N_{B, C}^{+}(s)$.

To summarize the situation, these are some facts about the situation we are in:

1. $C$ is an initial strong component of $D-s$.

2. $s$ has at least $f(k)$ out-neighbours inside $C$. We will denote this set of outneighbours by $S=N_{B, C}^{+}(s)$ for brevity.

3. Every cycle in $C$ contains at least two vertices of $S$.

4. By definition of the arc set $B$, for each vertex $u \in S$ there exists a subdigraph $D^{\prime}$ of $D-\{s, u\}$ with $\delta^{-}\left(D^{\prime}\right) \geq 1$ and $\left|V\left(D^{\prime}\right)\right| \geq k_{2}$.

5. $D-C-s$ does not have a subdigraph with $\delta^{-} \geq 1$ and size at least $k_{2}$, so some of the vertices of the subdigraph $D^{\prime}$ from fact 4 belong to $C$. Additionally, we had from fact 1 that $C$ is initial, so some cycle in $C$ must be part of the subdigraph of minimum in-degree at least one that we are looking for.

6. The objective is to show that there exists a cycle in $C$ that avoids at least $k_{1}$ vertices in $S$
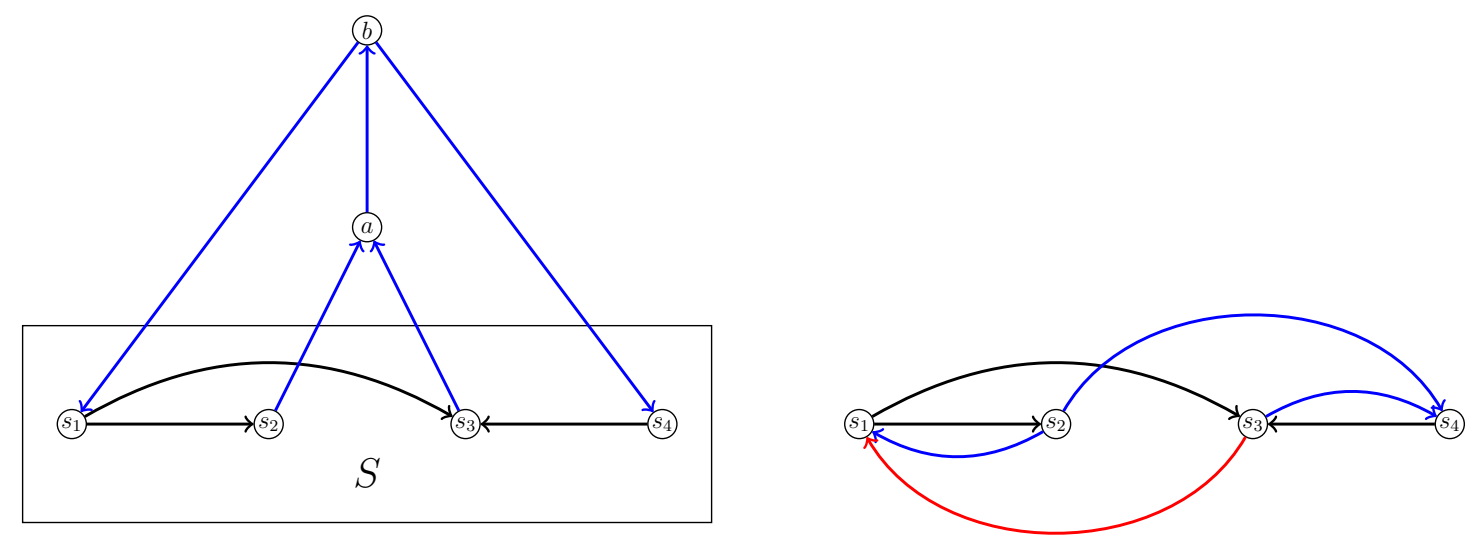

Figure 1: An example of the construction of $D_{S}$. Left: the strong component $C$ with $S=N_{B, C}^{+}(s)$ and $D\langle S\rangle$ shown inside the rectangle. Right: the final digraph $D_{S}$ with the red arc representing the path $s_{3} \rightarrow a \rightarrow b \rightarrow s_{1}$. The other new arcs are shown in blue.

Let the digraph $D_{S}$ be obtained as follows, starting from a copy of $D[S]$ : For each ordered pair $a, b \in S$, such that there is a path $a \rightarrow v_{1} \rightarrow v_{2} \rightarrow \ldots \rightarrow v_{l} \rightarrow b$ in $C$ where all $v_{i} \notin S$ for $i \in[l]$, we add the arc $a b$ to $D_{S}$, if it does not already exist. Note that, $D_{S}$ does not have parallel arcs. We use $d_{S}^{+}(u)\left(d_{S}^{-}(u)\right)$ to denote the out-degree (in-degree) of vertex $u$ in $D_{S}$.

Let $a, b \in S$ and note that since $C$ is strong it contains an $(a, b)$-path $P$. Let $\left\langle u_{1}, \ldots, u_{m}\right\rangle$ be those vertices on $P$ that are in $S$, listed in the order in which they are visited by $P$. By the construction of $D_{S}$ it contains the path $a \rightarrow u_{1} \rightarrow \ldots \rightarrow u_{m} \rightarrow b$ 
in $D_{S}$. Since $a, b$ were arbitrary vertices, it follows that $D_{S}$ is strong. With a similar argument and the fact that every cycle in $C$ contains at least two vertices of $S$, we can conclude that every cycle in $C$ corresponds to a cycle in $D_{S}$. We also have that a cycle in $D_{S}$ corresponds to a closed walk in $C$, so a cycle in $D_{S}$ that avoids at least $k_{1}$ vertices in $S$ is verification that there exists a cycle in $C$ which avoids at least $k_{1}$ vertices in $S$.

Let $g=g\left(D_{S}\right)$ denote the girth of $D_{S}$ and let $t=\min \left\{t^{+}, t^{-}\right\}$, where $t^{+}=\mid\{u \in$ $\left.D_{S} \mid d_{S}^{+}(u)=1\right\}\left|, t^{-}=\right|\left\{u \in D_{S} \mid d_{S}^{-}(u)=1\right\} \mid$. Now we apply Theorem 4 to $D_{S}$ (recall that this holds for both $t^{+}$and $t^{-}$). If $t=0$, then because $|S| \geq f(k)>2 k_{1}$ we have $g \leq\lceil|S| / 2\rceil \leq|S|-k_{1}$. Otherwise, by Theorem 4, we have $g \leq\lceil(|S|+t-1) / 2\rceil$. If $t \leq|S|-2 k_{1}$ we make the following calculation.

$$
\begin{aligned}
& t \leq|S|-2 k_{1} \\
& \Downarrow \\
& \Downarrow \\
& |S|+t \leq 2|S|-2 k_{1} \\
& g \leq\lceil(|S|+t-1) / 2\rceil \leq|S|-k_{1},
\end{aligned}
$$

implying that every shortest cycle in $D_{s}$ avoids at least $k_{1}$ out-neighbours of $s$ in $D_{S}$. Clearly we can find a shortest cycle in polynomial time. Hence we may assume that $t>|S|-2 k_{1}$. This implies that at least $|S|-4 k_{1}$ vertices have $d_{S}^{+}=d_{S}^{-}=1$. Denote by $T$ the set of these vertices and let $\bar{T}=S \backslash T$. Consider $D_{S}[T]$, it will consist of some vertex-disjoint induced paths (as $\bar{T} \neq \emptyset$, by the definition of $B$ ). Let $\mathcal{P}$ denote the set of these paths. Clearly $\mathcal{P}$ can be computed in polynomial time. Note that for each path $P=v_{1} \rightarrow \ldots \rightarrow v_{l} \in \mathcal{P}$, there is exactly one pair $a, b \in \bar{T}$ such that $a \rightarrow v_{1}$ and $v_{l} \rightarrow b$ are arcs in $D_{S}$. Use $a \stackrel{P}{\rightarrow} b$ to denote a path from $a$ to $b$ using the path $P \in \mathcal{P}$.

Suppose first that there is a path $P \in \mathcal{P}$ with at least $k_{1}$ vertices. We claim that there must be a cycle $W$ in $D_{S}$ avoiding $P$. Assume for the sake of contradiction that there is no such cycle. Then, because every vertex $u$ on $P$ has $d_{S}^{+}(u)=d_{S}^{-}(u)=1$, every cycle in $D_{S}$ must contain the entire path $P$. Thus removing any vertex $v$ of $P$ would destroy all cycles in $D_{S}$ and hence also in $C$. But because $C$ was initial, this means no vertices in $C-v$ could be part of a subdigraph with $\delta^{-} \geq 1$, and as we are in a case where $D-C-s$ does not have subdigraph with $\delta^{-} \geq 1$ and size at least $k_{2}$, we get a contradiction to the definition of $N_{B}^{+}$(since the arc $s v$ is in $B$ ).

Suppose now that every path $P \in \mathcal{P}$ has less than $k_{1}$ vertices. Recall that we have at most $4 k_{1}$ vertices in $\bar{T}$. We can represent $D_{S}$ by a directed multigraph $D_{\bar{T}}$ with vertex set $\bar{T}$ and with the $\operatorname{arcs} A_{\bar{T}} \cup A_{\mathcal{P}}$ where $A_{\bar{T}}=A\left(D_{S}[\bar{T}]\right)$ and $A_{\mathcal{P}}=\{a \stackrel{P}{\rightarrow} b \mid$ $a, b \in \bar{T}, P \in \mathcal{P}\}$. See Figure 2 , 

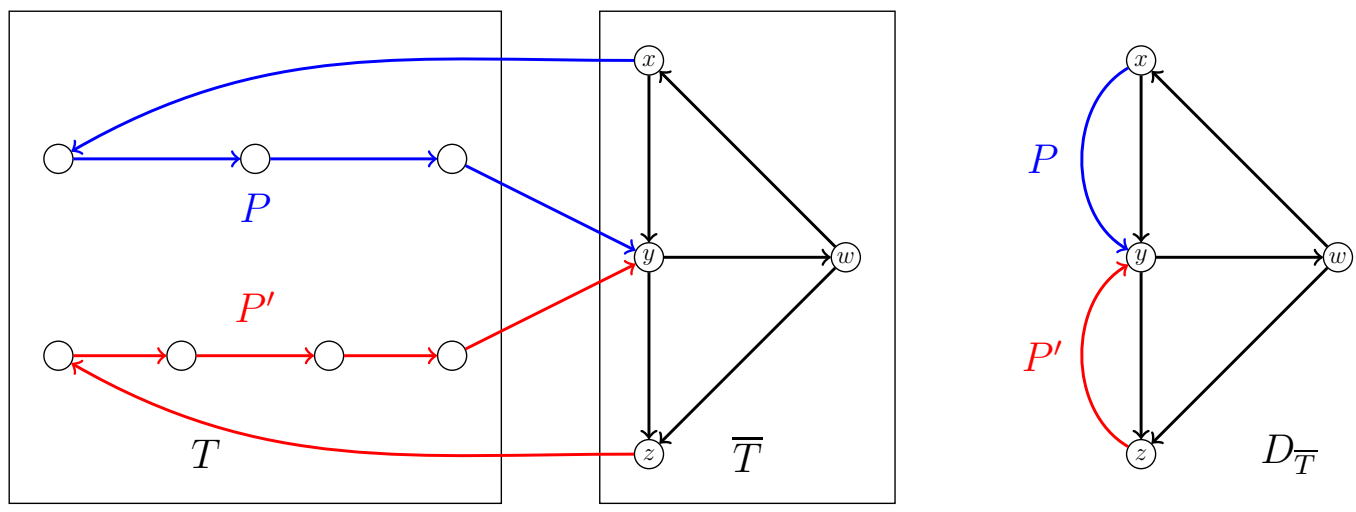

Figure 2: The left part of the figure shows an example of the sets $T, \bar{T}$, illustrating that $D\langle T\rangle$ is a collection of vertex disjoint paths. The right part shows the extra (coloured arcs that are added when we create the digraph $D_{\bar{T}}$.

For two distinct vertices $a, b$ of $D_{\bar{T}}$, let

$$
w_{a b}=\sum_{a \stackrel{P}{\rightarrow} b \in A_{\mathcal{P}}}|P|
$$

be the number of vertices in $T$ which lie on paths from $a$ to $b$ in $D_{S}$. Note that

$$
\sum_{a, b \in \bar{T}} w_{a b}=|T|
$$

As there are at most $4 k_{1}$ vertices in $D_{\bar{T}}$, there are less than $\left(4 k_{1}\right)^{2}=16 k_{1}^{2} \leq 16 k^{2}$ pairs. We also have that

$$
\begin{aligned}
|T| & \geq|S|-4 k_{1} \\
& \geq f(k)-4 k_{1} \\
& \geq 32 k^{3}+4 k-4 k_{1} \\
& \geq 32 k^{3}
\end{aligned}
$$

So it follows from (2) that there must be a pair $a, b$ that has

$$
\begin{aligned}
w_{a b} & \geq \frac{32 k^{3}}{16 k^{2}} \\
& =2 k \\
& \geq 2 k_{1}
\end{aligned}
$$

Let $\mathcal{P}_{a b} \subseteq \mathcal{P}$ denote the set of those paths that contribute to $w_{a b}$. Since each $P \in \mathcal{P}$ has $|P|<k_{1}$, we have $\left|\mathcal{P}_{a b}\right| \geq 2$ so if we choose $P^{\prime} \in \mathcal{P}_{a b}$ as one of these that uses the fewest vertices, then we always avoid at least $k_{1}$ vertices in the union of the other paths in $\mathcal{P}_{a b}$. Because $D_{\bar{T}}$ is strong, there is also a path $P^{\prime \prime}$ from $b$ to $a$ in $D_{\bar{T}}$ and $P^{\prime \prime}$ does not intersect any path in $\mathcal{P}_{a b}$. Hence $P^{\prime} \cup P^{\prime \prime}$ is a cycle that avoids at least $k_{1}$ vertices of $S$.

In conclusion we have shown that if we are in Case (2.1), then in polynomial time we can find a cycle which avoids at least $k_{1}$ vertices of $S$. 
Case 2.2. $\forall C \in \mathscr{C}:\left|N_{B, C}^{+}(s)\right|<f(k)$ :

As there are $h(k)$ out-neighbours of $s$ there must be at least $\frac{h(k)}{f(k)-1}=\frac{2 k \cdot f(k)}{f(k)-1} \geq 2 k$ strong components containing a neighbour of $s$. From the definition of $N_{B}^{+}$, we know that $D-s$ has a subdigraph $D^{\prime}$ with $\delta^{-}\left(D^{\prime}\right) \geq 1$ and at least $k_{2}$ vertices. We can build such a subdigraph that also leaves at least $k_{1}$ out-neighbours of $s$ for the outbranching as follows: Starting from an empty set $V_{2}^{\prime}=\emptyset$, iteratively add the vertices of a non-trivial strong component of $D-s-V_{2}^{\prime}$ to $V_{2}^{\prime}$ and use Algorithm 1 on $D-s$ with $S=V_{2}^{\prime}$ and $k=k_{2}$ to grow it. We repeat until $\left|V_{2}^{\prime}\right| \geq k_{2}$ (when adding a non-trivial strong component we may exceed $k_{2}$ ).

As $s$ has an out-neighbour in at least $2 k$ strong components and at most $k_{2} \leq k$ strong components are added to $D_{2}^{\prime}$ during this construction, there are still at least $2 k-k_{2} \geq k \geq k_{1}$ strong components, containing a neighbour of $s$, that can be used to form an out-tree from $s$ of size at least $k_{1}$. So we can obtain a subsolution in polynomial time.

Case 3. $\exists u \in V:\left|N_{B}^{+}(u)\right|>h(k)$ and $\exists ! r \in V: d^{-}(r)=0$ :

As we saw in Case 2, if a vertex $s$ has $\left|N_{B}^{+}(s)\right|>h(k)$ and every other vertex has indegree at least 1 , then we have a yes-instance. So the idea in this case is to start with $r$ and while $\left|N_{B}^{+}(r)\right| \leq h(k)$ contract a neighbour $u \in N_{B}^{+}(r)$ into $r$ and recompute $B$ and $N_{B}^{+}(r)$. If we reach $N_{B}^{+}(r)=\emptyset$ before contracting $k_{1}-1$ times, then we backtrack and try contracting another neighbour in $N_{B}^{+}(r)$, until we have tried all possible out-trees with $k_{1}$ vertices from $r$, similar to case 1. If we instead reach $\left|N_{B}^{+}(r)\right|>h(k)$ then the problem is reduced to case 2, and we have a yes-instance.

Because we reduce to Case 1 and 2 in polynomial time, this case is also solvable in FPT time.

So we have shown how to find the correct answer in all cases. We also argued that it was possible in FPT time in every case. This concludes the proof.

From the proof in Case 2 we obtain the following.

Corollary 6. Let $\left(D=(V, A), k_{1}, k_{2}\right)$ be an instance of the $\left(B^{+}, \delta^{-} \geq 1\right)-\left[k_{1}, k_{2}\right]$ PARTITION-problem, with $\delta^{-}(D) \geq 1$. Let $B, N_{B}^{+}$be defined as in the beginning of the proof of Theorem 5 and let $k=\max \left\{k_{1}, k_{2}\right\}$. If there is a vertex $s \in V$ with $\left|N_{B}^{+}(s)\right| \geq 64 k^{4}+8 k^{2}$, then we can find a good 2-partition in polynomial time.

The $\left(B^{-}, \delta^{+} \geq 1\right)-\left[k_{1}, k_{2}\right]$-PARTITION-problem is the analogoue of the $\left(B^{+}, \delta^{-} \geq 1\right)$ $\left[k_{1}, k_{2}\right]$-PARTITION-problem where we want an in-branching in one set of the partition while the other induces a digraph of minimum out-degree at least 1. By considering the digraph that we obtain by reversing all arcs we see that the following holds.

Corollary 7. The $\left(B^{-}, \delta^{+} \geq 1\right)-\left[k_{1}, k_{2}\right]$-PARTITION-problem is FPT.

\section{Remarks and open problems}

If we relax the condition of having an out-branching to that of just being connected, we obtain the following problem. 
(connected, $\left.\delta^{-} \geq 1\right)-\left[k_{1}, k_{2}\right]$-PARTITION

Input: A digraph $D=(V, A)$ and natural numbers $k_{1}, k_{2}$

Question: Is there a 2-partition $\left(V_{1}, V_{2}\right)$ of $V$ such that $D\left[V_{1}\right]$ is connected, $\delta^{-}\left(D\left[V_{2}\right]\right) \geq 1$ and $\left|V_{i}\right| \geq k_{i}$ for $i=1,2$ ?

Theorem 8. [1] The (connected, $\delta^{-} \geq 1$ )-[ $\left.k_{1}, k_{2}\right]$-PARTITION-problem (for fixed $k_{1}, k_{2}$ ) is NP-complete for general digraphs and polynomially solvable for strong digraphs.

Theorem 9. The (connected, $\delta^{-} \geq 1$ )-[ $\left[k_{1}, k_{2}\right]$-PARTITION-problem is FPT for digraphs with minimum in-degree at least 1.

Proof. First observe that if $D=(V, A)$ has minimum indegree at least 1 and $V_{1}^{\prime}, V_{2}^{\prime}$ are disjoint sets such that $\left|V_{i}^{\prime}\right| \geq k_{i}, D\left[V_{1}^{\prime}\right]$ is connected and $\delta^{-}\left(D\left[V_{2}^{\prime}\right]\right) \geq 1$, then we can easily extend this to a 2-partition $\left(V_{1}, V_{2}\right)$ of $V$ with $V_{i}^{\prime} \subseteq V_{i}, i=1,2$ where $D\left[V_{1}\right]$ is connected and $\delta^{-}\left(D\left[V_{2}\right]\right) \geq 1$. Hence it suffices to show that we can find a subsolution, if one exists, in FPT time. The proof of this is an easy modification of the proof of Theorem 5. We leave the details to the interested reader.

The theorem also holds for the analogous (connected, $\left.\delta^{+} \geq 1\right)-\left[k_{1}, k_{2}\right]$-PARTITIONproblem, for digraphs with minimum out-degree at least 1 .

\section{References}

[1] J. Bang-Jensen, N. Cohen, and F. Havet. Finding good 2-partitions of digraphs II. Enumerable properties. Theor. Comput. Sci., 640:1-19, 2016.

[2] J. Bang-Jensen and G. Gutin. Digraphs: Theory, Algorithms and Applications. Springer-Verlag, London, 2nd edition, 2009.

[3] J. Bang-Jensen and F. Havet. Finding good 2-partitions of digraphs I. Hereditary properties. Theor. Comput. Sci., 636:85-94, 2016.

[4] J. Bang-Jensen, K.V. Klinkby Knudsen, S. Saurabh, and M. Zehavi. The parameterized complexity landscape of finding 2-partitions of digraphs. Theor. Comput. Sci., 795:108-114, 2019.

[5] J. Bensmail. On the complexity of partitioning a graph into a few connected subgraphs. J. Combin. Optim., 30:174-187, 2015.

[6] D. Bokal, G. Fijavz, M. Juvan, P.M. Kayll, and B. Mohar. The circular chromatic number of a digraph. J. Graph Theory, 46(3):227-240, 2004.

[7] T. Feder, P. Hell, and C.S. Subi. Complexity of acyclic colorings of graphs and digraphs with degree and girth constraints. CoRR, abs/1907.00061, 2019.

[8] D. Kühn and D. Osthus. Partitions of graphs with high minimum degree or connectivity. J. Combin. Theory Ser. B, 88:29-43, 2003. 
[9] N. Lichiardopol. Vertex-disjoint subtournaments of prescribed minimum outdegree or minimum semidegree: Proof for tournaments of a conjecture of Stiebitz. Intern. J. Combin., Article ID 273416:1-9, 2012.

[10] N. Misra, G. Philip, V. Raman, S. Saurabh, and S. Sikdar. FPT algorithms for connected feedback vertex set. J. Combin. Optim., 24:131-146, 2012.

[11] J. Shen. On the girth of digraphs. Discrete Math., 211(1-3):167-181, 2000.

[12] M. Stiebitz. Decomposition of graphs and digraphs. In KAM Series in Discrete Mathematics-Combinatorics-Operations Research-Optimization 95-309. Charles University Prague, 1995.

[13] H. Suzuki, N. Takahashi, and T. Nishizeki. A linear algorithm for bipartion of biconnected graphs. Inform. Process. Lett., 33:227-231, 1990.

[14] P. van't Hof, D. Paulusma, and G.J. Woeginger. Partitioning graphs into connected parts. Theor. Comput. Sci., 410:4834-4843, 2009. 